\title{
Shared motivations, goals and values in the practice of personal science - A community perspective on self-tracking for empirical knowledge
}

\author{
Enric Senabre Hidalgo ${ }^{1}$, Mad P. Ball ${ }^{2}$, Morgane Opoix ${ }^{1}$, Bastian Greshake Tzovaras ${ }^{12}$
}

1. Center for Research and Interdisciplinarity, INSERM U1284, Université de Paris, Paris, FR

2. Open Humans Foundation, Sanford, NC, US

3. Center for Research and Interdisciplinarity, Paris, FR

\begin{abstract}
Some individuals do not limit their self-tracking efforts to passively collecting and observing gathered data about themselves, but rather develop it into forms of self-research and self-experimentation, also called "personal science". This type of N-of-1 research is relevant to the fields of personal informatics, patient-led research and social studies of science, but as a knowledge generation practice is still poorly understood. To fill this gap, we conducted 22 semi-structured interviews to investigate the intrinsic and extrinsic motivations of individuals engaging in personal science activities, as well as shared goals and values present in self-research communities. Our analysis is based on a conceptual framework that integrates previous approaches in self-research, as well as in connection with citizen science, the scientific ethos and cooperation in peer production. We identify how self-researchers seek to go beyond personal metrics about their health and wellbeing regarding data provided by wearables, are engaged over time by individual involvement in technology and scientific-related activity, and collaborate following similar goals and values when learning and sharing empirical knowledge with peers. In this sense, personal science can be understood as an example of a more participatory and inclusive scientific culture driven by self-reflection, critical thinking and openness.
\end{abstract}




\section{Introduction}

Self-tracking - collecting and observing data on one's body, life and self - has been widely studied in recent years. But self-tracking is not always limited to this passive engagement with the gathered data. Some individuals develop their quantified self practices into new forms of self-research and self-experimentation (Lupton, 2019; Neff and Nafus, 2016). Current modes of self-research can be traced back to early examples of self-experimentation in medicine (Weisse, 2012), but the notion of personal science has recently emerged as a framework to understand self-tracking in a wider context of empirical knowledge production (Heyen, 2016). The term "personal science" - originally coined by the self-experimenter Seth Roberts (2012) - has been defined as "the practice of using empirical methods to pursue personal questions" (Wolf and De Groot, 2020), and conceptualized as "N-of-1 research" (De Groot et al., 2017). Personal science can also be understood as science-based knowledge developed by citizens and laypeople (Heyen and Dickel, 2019). Recent studies on personal health science regarding Parkinson's disease (Riggare et al., 2021) have additionally pointed to ethical issues when considering the role of the researcher and the participant being the same person. Furthermore, Heyen (2020) has observed how self-researchers use scientific-like procedures to produce personal knowledge in their daily lives, pointing to an increasingly multifaceted picture of self-tracking beyond health-related topics. Other scholars have approached this phenomenon from alternative terms, such as "one person laboratory" (Christiansen et al., 2018), or as a cycle of iterative empirical inquiry for a personal science framework (Wolf and De Groot, 2020). In line with these perspectives, we explore the intrinsic and extrinsic motivations of "self-researchers" and how they lead to engagement over time, identifying shared objectives and values between practitioners. As a participant-led activity originated from self-tracking, personal science represents an emergent paradigm that can contribute new perspectives to the topic of the quantified self, especially in the fields of personal informatics, patient-led research and science and technology studies.

\section{Self-tracking and personal informatics}

Within the fields of personal informatics and Human Computer Interaction (HCI), there has been an increased interest in understanding user's tracking of personal data in areas such as health, sports, productivity and others (Jarrahi et al., 2018). This research expands on previous approaches such as "lifelogging" (Rapp et al., 2018), exploring how self-tracking tools can provide individuals with actionable self-insights to change behavior and improve their quality of life (Dijk et al., 2017). Epstein et al. (2020) show that personal informatics has mainly focused on self-tracking for health and wellness, and on identifying potential user needs. This has allowed to identify barriers in the user experience of personal data gathering and design improvements for self-tracking technologies (Li et al., 2010).

However, there has been limited personal informatics research into how to support individual interpretation and sense making to "transform numbers into meaning" (Rapp et al., 2018), while other studies consider how self-tracking tools strive to assist successful behavioral change (Rapp and Tirassa, 2017). On the other hand, HCI studies on self-tracking have rarely addressed its collaborative implications, except for some patient communities' knowledge sharing and do-it-yourself practices (Kaziunas et al., 2018). Although the study of collaboration in self-tracking from this field is relatively scarce, approaches such as "lived informatics" (Rooksby et al., 2014) or "quantified us" (Dijk and 
Ijsselsteijn, 2016) point to the importance of social and cooperative dynamics. Beyond motivations to improve health or wellbeing, other personal informatics studies also point to lack of scientific rigor in self-research practices, and how this can lead to inconclusive interpretation of results (Choe et al., 2014) or discouraging self-tracking (Eikey et al., 2021).

\section{The example of patient-led communities}

In patient-led research and online patient communities, self-tracking has also been explored regarding personal health data gathering and management (Almalki et al., 2015), and addressing the individual right to scientific activity and a more participatory scientific culture (Vayena et al., 2016). Considering it an opportunity for engagement and collaboration outside traditional researcher-participant contexts (Chrisinger, 2020), Riggare et al. (2019) identify how self-tracking offers Parkinson's patients a deeper understanding of chronic conditions, contributing to decision making regarding their own selfcare. In communities of cluster headache patients, it has been observed that group dynamics shape collective self-experimentation, including interventions, data analysis and treatment efficacy (Kempner and Bailey, 2019). Another example from patient-led research is the continuous glucose monitoring community, as active online groups of peer support characterized by openness, data altruism and mutual empowerment (Gavrila et al., 2019) This has sparked collective experimentations beyond the broader diabetes community (Grant et al., 2019), with practices of transparency and peer-support among self-trackers through group discussions and sharing of resources.

Other studies on patient-led self-tracking describe how social identity and interaction within communities drives co-creation, based on reciprocal trust and shared goals (Zhao et al., 2015). On the other hand, Ruckenstein and Schüll (2017) observe how the datafication of the health ecosystem is generating new power asymmetries and disrupting traditional regulatory and ethical research mechanisms, as something extrapolable to the "datafication of life" beyond clinical and self-care practices. Patient-led literature on self-tracking also focuses on the need of eliciting individual goals to avoid pitfalls like ineffective tracking routines or breakdowns in collaboration (Munson et al., 2020). Finally, another recent perspective from patient-led research studies (Vuolanto et al. 2020) points to how self-tracking patient communities usually try to apply scientific ideals of ethical conduct and rational scepticism for evidence based knowledge.

\section{From critique to knowledge value perspectives in social studies of science}

In the context of science and technology studies, Lupton (2019) identifies the agential capacities of self-trackers for achieving personal knowledge, awareness and problem-solving, in a context of "human-nonhuman assemblages" and in line with technology and data "mediated self-knowledge" (Jethani, 2015). Social sciences and humanities scholars have also explored potential harms derived from self-tracking practices: e.g. the impact of (self-)surveillance (Esmonde, 2020); "digital divide" perspectives (Régnier, 2018); or the biopolitics attached to this phenomenon (Moore and Robinson, 2016). Referring to the concept of "metric culture", Ajana (2017) links these critical perspectives to issues of power and control, and to questions of knowledge value and personal agency. In contrast, considering personal data-gathering practices outside such "data fetishist" critique, Sharon and Zandbergen (2017) 
describe ways in which self-trackers attribute meaning to their data, moved by self-reflection and as a communicative and narrative process. While there is scarce research into how users of wearable devices and apps subjectively experience self-tracking (Lyall and Robards, 2018), some studies approach the agency of self-trackers' to make sense of personal data beyond visualizations and algorithms pre-defined by market technologies, as a reflective and open-ended relationship with metrics (Kristensen and Ruckenstein, 2018). As proposed by Lupton and Smith (2018), enactments of self-tracking can be understood as an interrelation of motivations and capacity building for self-improvement - e.g. to achieve specific goals. Regarding the study of community contexts, other authors address the eclectic ways in which self-tracking leads to experimenting and learning through communication processes with peers (Pantzar and Ruckenstein, 2017), and how individual practices of data sharing are amalgamating and connecting around supportive places like the Quantified Self community (Sharon, 2017) or, more recently, the Open Humans platform (Trace and Zhang, 2019).

In relation to knowledge-generation cycles involved in self-tracking, previous qualitative studies identify how practitioners are potentially immersed in several tasks and roles required to gain insight from personal data. This includes appropriation of tools, observation of variables and interpretation (Lupton, 2019), in a flexible system of epistemological enquiry (Ruckenstein and Pantzar, 2017). From such perspectives, self-tracking represents a changing palette of "situated objectivity", aligning with initiatives for the democratization of science (Burnside et al., 2020). Personal research guided by self-tracking can be understood as a "missing link" within recent movements and paradigms such as "DIY science" (Ferretti, 2019) or citizen science (Hecker et al., 2018). However, with few exceptions (Dolejšová and Kera, 2017; Heyen, 2016), the connection between citizens "who do science" and self-researchers "experimenting science" is not yet commonly considered from similar perspectives, and literature on citizen science hardly refers to these individual research-oriented practices.

Considering the conceptual perspective of personal science, and recent advances and gaps from the fields outlined above on self-tracking and the quantified self, we delve further into key issues of individual agency, community implications and knowledge generation processes in this participant-led phenomenon. Our study contributes to this shift in perspectives, between extremes of self-tracking and public participation in science, by offering a conceptualized qualitative view on seemingly relevant motivations, goals and values related to personal science practices: What are intrinsic and extrinsic motivations for individuals engaging in self-research, and how do they evolve over time? How are these individual motivations related to shared goals, values and practices in the context of personal science communities?

\section{Study context and methodology}

Our study is situated in the context of the "Keating Memorial (KM) Self-research" initiative, which invites the development and sharing of self-research projects, co-organized by the Quantified Self (QS) and Open Humans $(\mathrm{OH})$ communities between February and July 2020. The KM honors Steven Keating, a patient-researcher who passed away in 2019, known for his commitment to self-experimentation and promoting access to patient data. As part of the KM, weekly self-research chats open to personal science practitioners are organized for sharing and discussing preliminary ideas, results and protocols. Attended on average by 14 people monthly, these calls follow a self-organising governance format in which participants decide the agenda at the beginning of sessions, based on their updates on ongoing or potential 
projects. Examples of projects shared relate to the self-study of sleep quality, blood glucose, essential tremors, diet effect on mood, and noise sensitivity. The KM culminates in a yearly event to present and discuss self-research results in an open seminar. In its first edition, this included self-research on hay fever symptoms, cardiac arrhythmia, transition with testosterone and mental contrasting for well-being. This setting provides an opportunity to gain knowledge about motivations, goals and values in personal science.

\section{Participant enrollment and data collection process}

Data were collected by two authors (ESH and MO) through semi-structured interviews. A list of potential study participants from the KM initiative was established after observing their activity on community forums and calls. Initial interviewees were selected based on the following criteria: (1) Having conducted or attempted to conduct a personal science initiative; (2) Having joined at least two OH community calls; and/or (3) Having participated in QS public forums or OH Slack channels. Two researchers (BGT and MPB), familiar with the community as self-researchers themselves, verified that identified individuals were active members. Additionally, interviewed participants were asked if they knew other self-trackers who might be interesting to participate in our study. This snowball sampling approach expanded to participants active in self-research or with personal science projects outside the KM, serving as contrast in our analysis. All interviewed participants gave consent in line with the ethical approval by the Institutional Review Board (IRB) for this study.

Interviews were conducted via video-conferencing and based on a list of open-ended questions (Annex 1: https://zenodo.org/record/5543494), separated into key sections around participation and collaboration in personal science. The semi-structured interview format allowed participants to add additional prospects and provided opportunities for interviewers to ask questions beyond initial interview prompts. Recruitment stopped when a saturation point was reached, and interview recordings were automatically transcribed to text and subsequently manually corrected to remove transcription errors.

\section{Conceptual framework and codebook for interviews interpretation}

To analyze the interviews, we developed a conceptual framework (Figure 1) based on previous studies on motivations in citizen science (Jennett et al., 2016), the inquiry cycle of personal science (Wolf and De Groot, 2020; Heyen, 2016), the scientific values or ethos of the Mertonian norms (Merton, 1973) and collaboration in peer production (Spaeth and Niederhöfer, 2020). This latter focus on peer production is relevant to take into account other open, digital-mediated collaborative practices with social and technological implications. The final framework consists of five main non-exclusive categories: (1) Improving personal conditions; (2) Enjoying data, tech or research activity; (3) Extrinsic motivations; (4) Contributing to empirical self-knowledge; and (5) Sharing goals and values with peers. 


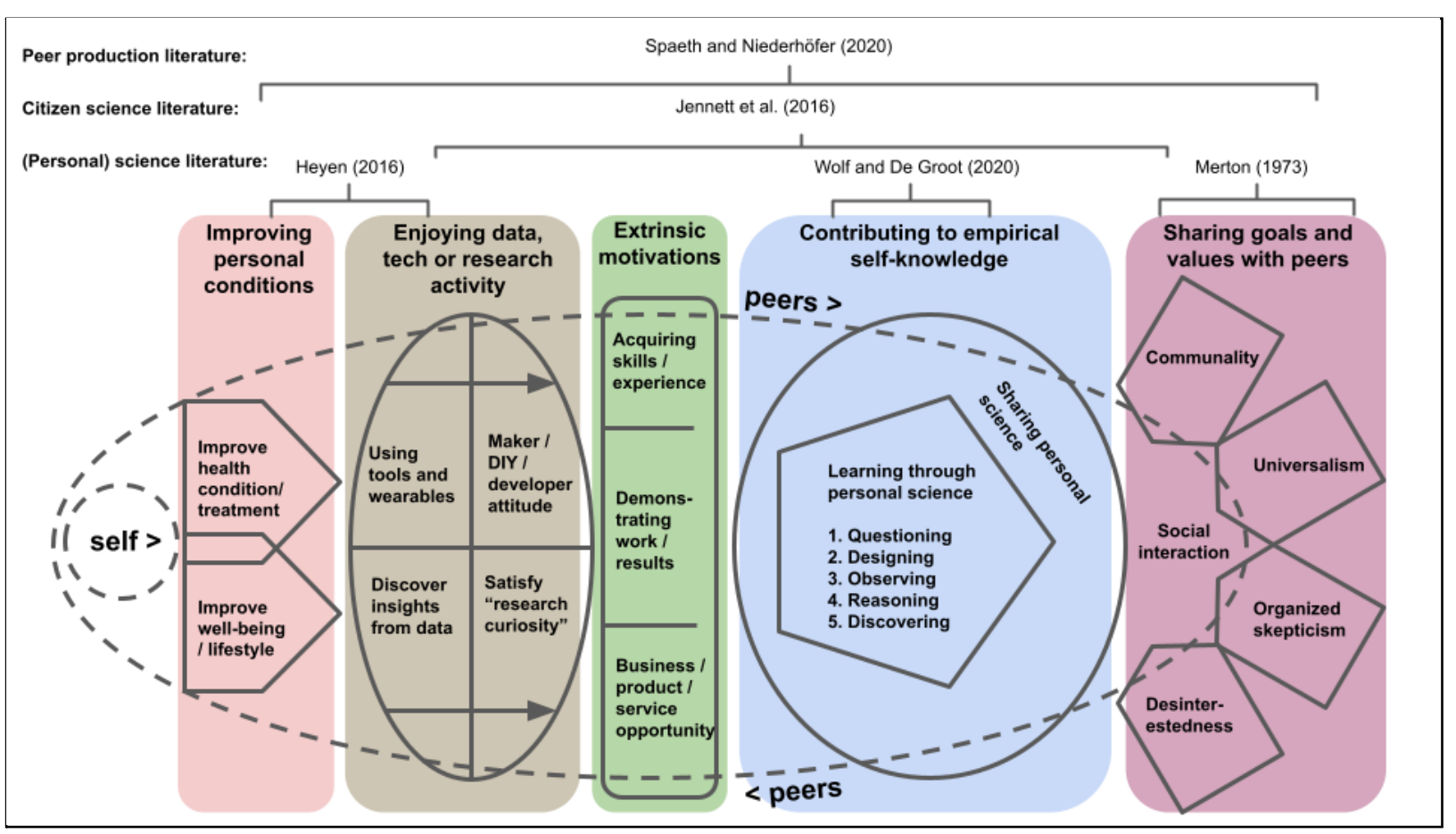

Figure 1: Conceptual framework for the study of motivations, goals and values in personal science

We iteratively developed a codebook alongside this framework to analyze the interviews. An initial codebook, designed by all authors, was tested for reliability and validity by coding the first interview and subsequently refined until agreement was reached. Its final version was used to code selected excerpts from the transcripts of all interviews in Taguette (Rampin et al., 2021), using the framework's main categories. This coding was performed by ESH and MO, with tags being assigned following discussion and reaching consensus between them. Each coded excerpt was then assigned to one interpretative sub-code (16 in total) within the assigned category (Table 1). The sub-coding was performed independently by ESH and MO and Intercoder reliability (ICR) was assessed using Cohen's Kappa. For sub-codes in categories 1 to 4, BGT acted as tie-breaker of conflicting assignments. Given the complexity of category 5 and correspondingly low IRC, all sub-codes were collectively discussed by the authors and sub-codes assigned on a consensus basis.

Table 1: Codebook with categories and subcodes for the interpretation of interviews

\begin{tabular}{|l|l|}
\hline Categories/codes & Specific subcodes \\
\hline 1. Improving personal conditions & $\begin{array}{l}\text { A. Improve specific health condition / treatment } \\
\text { B. Improve lifestyle / general well being }\end{array}$ \\
\hline 2. Enjoying data, tech or research activity & $\begin{array}{l}\text { A. Using tools and wearables } \\
\text { B. Discovering insights from data } \\
\text { C. Maker / DIY / developer attitude }\end{array}$ \\
\hline 3. Extrinsic motivations & D. Satisfying "research curiosity" \\
& $\begin{array}{l}\text { A. Acquiring skills / experience for professional career } \\
\text { B. Demonstrating work / results in a specific area }\end{array}$ \\
\hline
\end{tabular}




\begin{tabular}{|l|l|}
\hline & C. Business / product / service opportunity \\
\hline 4. Contributing to empirical self-knowledge & $\begin{array}{l}\text { A. Sharing personal science } \\
\text { B. Learning through personal science }\end{array}$ \\
\hline 5. Sharing goals and values with peers & $\begin{array}{l}\text { A. Social interaction } \\
\text { B. Communality } \\
\text { C. Universalism } \\
\end{array}$ \\
& $\begin{array}{l}\text { D. Organised skepticism } \\
\text { E. Disinterestedness }\end{array}$ \\
\hline
\end{tabular}

\section{Results}

The majority of the interviewees (18) participated in the KM, and were also part of other QS communities. 4 participants were recruited via snowball sampling, 3 of which were from outside any similar community and 1 participant only involved in the QS community, allowing for some heterogeneity of interviewees with different perspectives and experiences. Most participants (14) were from the United States, 3 from the United Kingdom and 1 from Switzerland, Sweden, the Netherlands, Canada and Spain each.

The interviews resulted in a final set of 269 transcribed excerpts being coded. ICR for the independent sub-coding of excerpts following the codebook categories 1-4 was 0.82 according to Cohen's kappa, where values between 0.80 and 0.90 are considered strong agreement. As Cohen's kappa for category 5 was only 0.22 , a consensus coding was made through discussion among authors.

Nearly all the self-researchers interviewed referred to a combination of the five main categories of analysis. In all cases, at least 3 categories were applied to each interview, and the majority of interviews (18) had excerpts codes from 4 of the 5 codebook categories (Figure 2). 


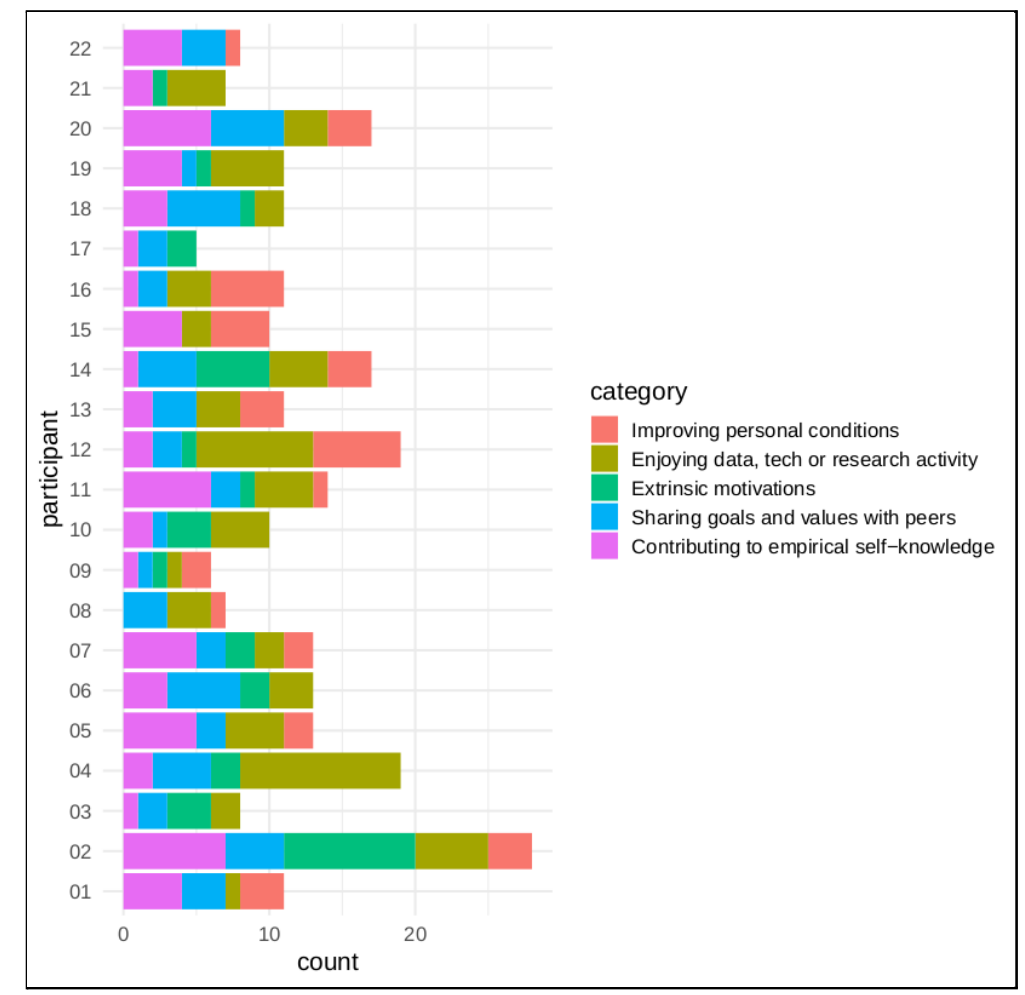

Figure 2: Distribution of codebook categories in interview excerpts.

We provide an open data set containing the details of all the excerpts processed and coded for our study (Annex 2: https://zenodo.org/record/5543446), with previous agreement of all the interviewees and in compliance with privacy and anonymity requirements.

\section{Initial motivations for improving health conditions and well-being}

Consistent with previous research (Schüll, 2018) we identify that early motivations for self-researchers relate to personal goals for solving specific health conditions, in the case of patient-led research practices, or to improve well-being in other cases. This highly personal motivational aspect, not usually considered in citizen science or other domains of participatory research, is specific to personal science (Lupton, 2014), and coincides with intrinsic motivations of "own use value" identified in the study of peer production (Spaeth and Niederhöfer, 2020). Regarding this type of motivation, patient-led self-researchers mentioned a desire to gain knowledge on personal, medical conditions. Similar motivations have previously been identified among patient-led self-tracking to self-manage a given disease or health condition (Gimpel et al., 2013). Participant \#16, who experiences chronic sleep disorders, expressed such interest in reference to solving a concrete health problem:

"My journey I think is a common one within this community, and that's when I was most interested and most active, I had a specific problem I was trying to solve for myself. For me that was the quality of my sleep. So I had lots and lots of self-tracking projects related to tracking both my sleep itself and the subjective quality of the sleep, and how I felt during the day". 
From a complementary perspective Participant \#22, who started to study symptoms and treatments regarding a chronic medical condition through a self-developed app in collaboration with other patients, refers to a motivation driven by personal unawareness and missing solutions for people who decide to start this type of self-research:

"I've been trying a shitload of different treatments, regular ones, alternative ones, and everything, and I never kept track of it. And then after eight years, you basically find out that you have no idea because you have tried so many things".

Significantly, these types of patient-led motivations seem to also trigger additional areas of inquiry, with individuals starting to track additional health-related variables in parallel, based on practical knowledge gained in previous self-research. For example Participant \#15, who practices personal science in relation to Parkinson's disease, expresses this tendency regarding COVID-19:

"Very recently I've been tracking Covid because I got Covid infection a couple weeks back, which I have been tracking as well. So that's my most recent project, but that was very much building on what I had learned from tracking over the years. I've learned so much from people tracking their conditions out there, in other areas. Although for myself, it's been mainly Parkinson's".

Another illustrative observation regarding this combination and somehow concatenation of intrinsic motivations, was made by Participant \#20, whose self-research on continuous glucose monitoring led to a DIY community of people with diabetes, and whose case represents another example of patient-led community research (Gavrila et al., 2019). Although this self-researcher also practices self-tracking regarding general physical and sport activity with a wearable, the interviewee made clear a sense of urgency and need to self-experiment which goes beyond usual perceptions on "quantified selves" (Kaziunas et al., 2018):

"It's weird as a person with type 1 diabetes to talk about self-tracking because from the moment one you're tracking, you have to stay alive. So self-tracking isn't really voluntary for people with diabetes. It's kind of a weird concept. So I've always been self-tracking but I think really where I dovetailed into more of this concept of self-research and researching and experimenting was about the time that I got frustrated with the tools that I had because I could not do the experimentation that I wanted to".

Among self-researchers who are originally motivated by improving their well-being or lifestyle, we also find clear parallels with other studies on self-tracking, identifying a combination of having a better lifestyle and well-being with the aspiration to be "a better self" (Ruckenstein, 2014). Participant \#05, who developed a personal research project on sleeping heart rate using Apple Watch during the first edition of the KM, exemplary expresses this type of initial motivation for self-research:

"I've always been healthy, but I'd like to sleep better and feel better and live longer, and all those sorts of things. So I don't consider myself to be anything special in that regard. I think everyone would like to be healthier and happier and live a better life. I guess maybe I'm a little bit more 
focused on doing it in a more rational way, and being a little bit more deliberate and maybe systematic about how I do that".

Another motivation mentioned by different interviewees is to get a deeper knowledge beyond what is provided by popular wearables and tracking apps, in a sort of subtle change of interest trying to go beyond "mere self-tracking", which reflects how the informational affordances of these tools may not sustain long-term activity (Jarrahi et al., 2018). Participant \#12, a self-researcher outside the QS and OH communities, refers to the time and effort of previous practices, tracking data manually over long periods of time, and how this can affect motivation:

"I still have memories of calorific content of certain foods that are like in my long-term memory. I mentally know, like I add up during the day how many calories I've eaten and where I'm at. But I find tracking it really boring on an app, I've tried a few nutrition tracking apps and I just find I get really bored of inputting it and it quickly loses interest in doing just that".

A majority of participants mention curiosity triggered by data gathered via wearables. We interpret this as a sign of long-term engagement with observational and explorative approaches, moved by a recurrent use of these tools and the quantitative information they provide (Choe, et al., 2015). For example, Participant $\# 14$, who among other things explores patterns of weight loss between running and cycling, mentions additional self-experimentation in the moment of the interview:

"I first stopped with coffee and around a year later I just stopped alcohol intake as well, to see what happens. Because I was already seeing in my Oura data that it was impacting my heart rate, and my heart rate variability during sleep, quite a lot".

Our results seem to confirm that self-related motivations are a key feature in personal science - as with self-tracking, c.f. "selfhood" in Lupton (2014) -. Furthermore, we find self-researchers commonly describe expanding or shifting to new self-related focuses over time (i.e. beyond original motivations related to health conditions or well-being), so new topics of motivational focus emerge in an interrelated manner, rather than independently. Relatedly, we find interests are not limited to personal metrics and interpretations provided by third parties (Ajana, 2020); self-researchers seek to go beyond these, often to rigorously investigate an initial question or concern. Analogous to academic research, the process of investigating one question tends to spark new, related research questions for participants, leading to on-going motivations over time.

\section{Reinforced motivations by enjoying data, research and tech-related activity}

Reinforcing motivation and engagement over time for non-expert involvement in scientific-related activity is critical in the domain of citizen science (Rotman et al., 2012). In the case of personal science, this connects with the second category of analysis of our framework as adopted from Jennet et al. (2016), through which we observe that the majority of self-researchers we talked to express enjoyment and involvement in research-related activities, like data gathering or using digital tools, as well as in other stages of personal inquiry. 
Enjoying data and "tech-related" activities (like customizing tools, combining data sources or plotting results), which imply practical knowledge needed for working with self-tracking technology, seems to be a relevant source of motivation for several interviewees, similarly to "fun" motivations for involvement in peer production (Spaeth and Niederhöfer, 2020). This can be seen as a form of non compliant, creative agency in extreme practices of self-tracking (Kristensen and Ruckenstein, 2018). An example is expressed by Participant \#04, who explores the use of new wearable technologies and other domestic sensors, and is interested in how these tools can be combined for new personal data visualizations. In this case the enjoyment is connected to several questions the participant is trying to solve about personal mood, attention and mental focus:

"I'm definitely excited about going up in the direction of using these different sensors, to extract those kinds of meaningful features. Like how far behind your normal routine are you, or what type of day are you having those kinds of things".

As "early-adopters", this acquisition of technical-related knowledge is a recurrent motivation for interviewees who have technological skills and express their continuous engagement as a sort of "hacker attitude" unfolding iteratively, as already identified by Sharon and Zandbergen (2017) in alternative self-tracking practices. Participant \#08 referred to this attitude in relation to a preference for passive tracking instead of actively logging data via apps or other tools, in this case practicing self-research for preventing hay fever and pollen allergy:

"So anytime there was a sensor tool that came along, because of its new capabilities, I was on to it, either crowdfunding it or building it myself from components. The point is that I'm more on the quantitative devices sensors than doing like the hay fever log of how I feel, because all my research on the brain says you can't really trust yourself".

Participant \#16 expresses also this type of motivation driven by "tech-related" enjoyment, in relation to open collaboration and re-use of other self-researcher's innovations, pointing to the limitations of “one-size-fits-all” tools (Kristensen and Ruckenstein, 2018):

"People end up writing tools that are specific to themselves and their problems. Certainly that has been my own. I think there are probably examples where people took the things I made and used them for their own purposes, in their projects. And I've taken other self-tracking tools that people made and used them for my purposes. And a lot of it is so individualized that it can be hard to make a general tool".

On the other hand, regarding continuous glucose monitoring, Participant \#20 considers the important motivation of developing and sharing tools as a starting point:

"When I talk with other people about self-tracking, their self-research or what they could do, I point out to them that building some of those tools to track or capture the data could then lead to that self-research, and a furthering of the research in that area. But it starts with having those tools". 
This key motivation of exploring new technologies leads some self-researchers who are non-patients to appropriate open source tools developed for medical use, like continuous glucose monitoring devices, blood test kits or electroencephalography sensors. Significantly, this type of appropriation of tools by non-patients in the context of personal science can be understood as an emergent "ecology of testing" (Marres and Stark, 2020), which has brought opportunities for coordinated participant-led research, like in the project "QS Blood Testers" (Grant et al., 2019). Participant \#11, refers to this as a general tendency:

"People are always looking for the next exciting gadget, maybe a blood test or other thing that can be tracked. Blood sugar tracking was very exciting, but now people are thinking maybe this other thing that can be other blood biomarkers that can be tracked continuously".

Engagement in self-research activities driven by enjoyment and curiosity relate significantly to additional transversal motivations for a better understanding of oneself's body, behavior or activity, and in this sense to questions of personal agency and reflexivity (Couldry and Powell, 2014). As reflected by Participant $\# 02$, who combines different sources of data for personal questions on well-being and quality of life:

"One of the things is curiosity, and personal self-discovery in a sense. We are on the path of self-discovery by means of this data and these experiments, so we can get to understand our bodies better. And we all have this, not just an open mind, but a growth mindset".

In this sense, continuous engagement in self-research to satisfy research curiosity about oneself, moving beyond an interest in data gathering tools per se, is another key aspect in how participants enjoy research-related activities, learning by practice about ways to generate and integrate "mediated self-knowledge" (Jethani, 2015). Starting from personal interests and questions connected to the motivations described above, several interviewees mention joyfulness and playfulness in connection to being involved in research-related activity, like described by Participant \#15:

"It's very much driven by joy, by having fun, by enjoying what to do. And once you find the answer to your personal question you don't need to keep continuing".

However, all self-researchers we interviewed kept different lines of personal research, regarding their original questions or concerns but also in connection to new ones. Another reason for this continued and iterative engagement seems to be connected to a common type of approach when starting to do personal science, which in many cases is observational and based on a first oversight of relevant data for a phenomenon during initial phases (Lupton, 2019). Participant \#19, one of the self-trackers who were contacted through snowball sampling, representing a perspective from the margins of the self-research community of this study, exemplifies this initial "inductive" approach in what constitutes a relatively extreme example, referring to the practice of regularly adding data-gathering mechanisms for daily activities and behavior:

"So basically I want a clear picture, minute by minute, of myself, what I'm doing and where I am. My goal is to passively track most of it. So my phone is tracking my location, even my indoor 
location, which room I'm in. I want to connect all of that together by data wrangling, so when I have enough data, then maybe I can learn from my patterns, my habits".

By contrast, as we will see later in more detail, this points to a general observation that personal science practitioners, when engaged in community activities for sharing and discussing experiences, tend to be more intentional, focused on specific questions and ways to obtain research results. Participant \#06, who participated in the KM initiative presenting a self-research project, reflected on this seemingly important aspect of research focus and interventional approach:

"The deadline was definitely motivating for getting something finished, where if not I think that I could have kept doing it for a really long time. You just think that there are like twenty things at once that I want to improve in my project. And so at some point you just have to wrap up some elements of it, and that was a really great motivation".

This type of intrinsic motivation stands as a clear example for the majority of interviewees active in the studied community, based on an experience accumulated over time when sharing their approaches or results with other self-researchers. In a wider sense, this motivation also relates to the background, personal history or mindsets from participants, which in different cases can be traced back to early research-related curiosity, like in the case of Participant \#07, who explored the effect of low-carb ingredients on blood sugar during the KM:

"I like experimenting things, and prior to doing self-research on essentially medical topics, I did lots of home chemistry experiments, and liked to build 3D printers and work out how to do testings".

In this regard, it is important to highlight that for some researchers such early interest in science and research did not have to do with current wearable technologies or sophisticated data-gathering tools, coinciding with self-trackers interviewed in previous studies (Lupton and Smith, 2018). An example of this is Participant \#12, who among other things tracks menstrual cycles:

"I've always done it on paper. I guess I don't know if that was really a conversation with my mom, who said you should track or you should just write it down. [...] I'm very digital anyway, but giving up the paper, because I've done that for so many years on paper, it was strange. But I do menstrual cycle tracking on an app now".

\section{The minor role of extrinsic motivations for doing self-research}

We find relatively few examples of interviewees influenced by the possibility of advancing on their academic or professional careers, pointing to a relatively minor role of extrinsic motivations in personal science. Some participants who are in parallel developing their PhDs on related topics mention this type of motivations as a relevant factor in their engagement with self-research, like expressed by Participant \#14: 
"[The $\mathrm{PhD}]$ has given me a lot of skills that I am also using as a quantified selfer, which is why I've recently published a little bit more on that. I just like it a lot, and what's kind of cool is that my $\mathrm{PhD}$ topic overlaps with self-tracking”.

Other interviewees mention the challenge of disseminating results from their projects in academic circles or journals (De Groot et al., 2017), like in the case of Participant \#07:

"In my career I have been doing a lot of things that then get published in papers, so it's just something I'm used to. I think that the trick is, normally, that the way I would analyse things would be collecting a lot more measurements with a lot more conditions, to write up like a reasonably thorough paper. To do that would either take an incredibly long time by myself, or I just have to have other people".

However, an overlap of intrinsic motivations with extrinsic ones in connection to professional activity is relevant for some self-researchers in determining why and how they proceed, as described by Participant \#06:

"I can do it on my own as a fun experiment, and I could also try to do it at my job or vice versa. So like I'll use a tool at work and I'll be like 'well this tool is actually pretty useful, I could use it for my own [self-research]'. So there's kind of that synergy that happens".

Another example, in this sense, is reflected in the conversation with Participant \#07, who had recently changed to a new job when interviewed:

"Before there was no connection between what I was doing in my job. Now there's a little bit, and one of the things that we're working towards is all sorts of small molecule protein and hormone diagnostics that will make it easier to do at home. [...] Or anything else that currently is hard to do at home testing for, if we can make that possible then there's a connection. Maybe I can sort of connect the two bits of my work".

These and other few participants seem additionally motivated by the possibility of acquiring new skills and expertise, either to support a "traditional" academic career or for advancing in their professional development.

Another extrinsic motivation factor, identified in even fewer interviews, relates to a parallel interest in how the medical and high-tech industries are constantly exploring and developing new data gathering tools and N-of-1 approaches, which dates back to the origins of the QS community (Ruckenstein and Pantzar, 2017) as described by Participant \#03:

"This area that you could also call 'very personal computing' was growing as a technology sector, and there was a lot of investment coming into it. So at the [QS] meetings we started to have some people getting up and speaking but they weren't really talking about their own personal experience, or about learning about themselves using these technologies". 
Participant \#11, in this sense, considers this possibility not as central but as an additional motivation for being involved in self-research:

"I'm also looking if there's any business opportunity. Like if you could just start a company if suddenly there was a huge demand, like from doctors who want to have a nice dashboard where they can look at their patients data... That would be an amazing opportunity, to build such a service".

\section{Common goals for learning and sharing empirical knowledge}

Relating to intrinsic motivations for engagement in self-research over time, the majority of participants refer to an overall importance of community processes and communication (Sharon and Zandbergen, 2017), openly sharing the different stages of their deductive or inductive approaches in order to get feedback and learn from peers. Coinciding at different levels with the incremental stages of each personal science process (Wolf and De Groot, 2020), participants are significantly motivated by being part of a research-oriented community, as summarised by Participant \#06:

"This is a nice place where you're motivated, you're kind of incentivized to share things as you're going, and that kind of opens up the discussion and I think also involves more people. Like when I presented my preliminary stuff [on a self-research topic], there was a really insightful discussion".

This "double-sided" factor of sharing and learning, in relation to motivations, is constantly identified through the conversations with self-researchers active in communities, like in the case of Participant \#11:

"The main motivation of sharing is just because I have been benefiting from reading about things other people have done, like the blood sugar tracking. [...] By following other people doing experiments I learned you can get these kinds of devices and how they work. So then when I do it, if I feel there's anything I learned in doing it, it's nice to share that too".

Community coordinated activities like the KM seem to contribute to building a sense of belonging, reinforcing participant's motivations to learn by sharing. These otherwise disconnected self-research practices find a common context and a shared framework for transparency and accountability, in which individual knowledge "produced in a methodically controlled manner" (Heyen, 2019) can benefit newcomers. Like expressed by Participant \#10, who recently started to join community meetings when interviewed, in this case interested in mood tracking and daily journaling:

"I read some books about self-research. I want to go more into it, I feel like I'm a beginner compared to all the other people attending these calls. I am now deeply interested in knowing more about myself scientifically".

Or regarding the incremental satisfaction of research curiosity expressed by Participant \#07, as another example: 
"Over time I got very interested in trying to understand this stuff sort of more rigorously and more quantitatively. And so I wanted to start doing more careful experimentation, really understanding a lot of things that were kind of just like lore or just things people kind of passed around in the community".

This learning process also comes by trial and error, implying that self-researchers expect to share failures, unsuccessful or non-concluding results from self-tracking (Choe et al., 2014), although as considered by Participant \#05:

"My results will not be as robust, but on the other hand, I can do a hundred experiments in the time that it takes your famous scientist to do one. 99 of my experiments will end up being irrelevant and wrong. But there's one that I find might be great, and that's always been the real appeal to me".

The majority of participants refer to community discussions and activities as an opportunity to discover each other's practices non-judgmentally and as a collective self-improvement process, when possible going beyond the data gathered to also understand the techniques and protocols ideated by other practitioners, regardless of their research topics. Another reflection from Participant \#15 points in that direction, regarding the importance of sharing and learning:

"It has to be disseminated and to be ethical. It has to be a value for society. It's a two step process. So in a way, as a personal scientist, I think you have a responsibility to also disseminate your findings, but maybe more importantly disseminate your methods".

These knowledge sharing practices are very relevant for the motivations, goals and expectations of a majority of self-researchers interviewed, and perceived accordingly in connection to values of openness, mutual trust and respect for the ideas or practices of peers (Zhao et al., 2015; Spaeth and Niederhöfer, 2020). This motivation for sharing usually refers to a common interest in scaling up research, for example involving more participants by opening up self-research data for others, as considered by Participant \#22 regarding the community of patients this interviewee is part of:

"I found out that I had no real knowledge or understanding of all the treatments I did. And I couldn't say what was effective or a little bit effective, you know. So I realized I needed to collect data, but I also realized that if I only looked at my data then everything I saw could be a coincidence. So then I got the realization that the only way that I could understand this as an individual person, and how it might change through time, would be compared to others, and this could only be done by joining data with others".

As a derived observation, examples of successfully scaling up practices beyond participants' aims (that is, successfully evolving in that direction) were only identified in interviews with patient-led self-researchers, who usually share, according to Participant \#20, similar goals:

"I'm sharing what I did as a hypothesis and here's my results, and you just want to casually compare with other people, kind of n-of-one to n-of-one. Some people then take it to the next 
level and say 'I actually want to study this so I'm going to specifically go out and ask people', and kind of facilitate a casual study. And so they kind of become a researcher there, where they're at least even informally and anecdotally gathering evidence from other people and validating that hypothesis for a subset of the community".

Something that proves to be usually complex and difficult, as expressed by Participant $\# 07$ :

"I had one success where we studied the effect of hot showers basically on blood glucose, which was brilliant. But other than that, I've just had a real challenge getting anybody to join in on things. I've had people now and again expressing interest, but they always drop out before we get anything done".

However, this general interest in scaling up constitutes a strong motivation for a substantial proportion of interviewees, which refer to aggregating similar data from other self-researchers (Nafus, 2019) or making their work or skills for developing tools available to peers (Kaziunas et al., 2018). Participant \#21, another participant contacted through snowball sampling, expresses this in relation to an interests beyond daily self-tracking via wearables:

"Right now I've been doing more analysis but to actually build useful tools for other people to use is what I'd be more interested in".

The social dimension of personal science motivations, when developed openly online and in a community of peers context, reinforces key factors for engagement over time for participants, who usually share their research processes and findings moved by a perception of common goals. We also observe that sharing of self-research practices happens largely in unstructured ways, like expressed by Participant \#13 in relation to exploring mood and personal relations:

"There's a lot of areas I want to go and that would be zoomed in but right now I'm kind of wondering what devices I need and how much data I need to have, and how do they connect. I'm not quite there at the research question".

Several examples reflect this lack of clear protocols and questions in research-related phases beyond data gathering, where complexities in detail and approach may vary. In several cases this refers to a type of personal commitment that has to lead with day-to-day limitations and the time-demanding practice of rigorous self-tracking (Neff and Nafus, 2016), as remembered by Participant \#20 when started to practice self-research:

"Also to try to figure out how to do research from a non-traditional perspective, you know, I wasn't at an academic institution. I was not at a company, I had no funding. It was what I did with my willingness and limited bandwidth in addition to my day job, to help answer these questions".

Lastly, another kind of observation has to do with the issue of connecting with academic researchers, experts and professionals outside the community. This refers to publishing results or sharing them with medical doctors, which in general is seen as problematic and complex (Piras, 2019). While several 
interviewees mentioned their activity on social media or personal websites, the further step of sharing self-research in academic publications is not relevant for non-academic participants, like considered by Participant \#11:

"Writing blog posts and giving talks is something it is about as much effort as I'm willing to put into that. I'm not going to write any academic papers or write any bestsellers or anything".

Whereas in relation to sharing self-research with health professionals, according to Participant \#15, they usually involve the same "epistemic" challenge (Fricker, 2007):

"Doctors are hesitant to engage in discussions on self-tracking with people. [...] I know for a fact that this attitude among doctors is very, very common, and there's actually an interesting concept that you might want to look into regarding this: epistemic injustice".

\section{Transversal values aligned with social and scientific practices}

Lastly, we investigate how some values expressed explicitly and implicitly through the majority of the interviews seem to drive individuals long-term activities in a community of peer's. On the one hand, as also seen in citizen science (West and Pateman, 2016), we observe a recurrence of social motivations in the desire to meet and discuss with like-minded people, as expressed by Participant \#06:

"I really like the community part, so that's not missing. I feel pretty involved with that. I think that if I had not joined during the Keating Memorial project, it would have been harder to join. There was just a lot of commonality, everyone had the same kind of baseline. It's kind of like a club".

On the other hand, we identify that community values have a relevant connection with the Mertonian norms or ethos for shared and ethical scientific practices, as previously suggested by Heyen (2016). These in turn align with the sharing culture of peer production, as suggested by other scholars (O'Neil et al., 2020; Dulong et al., 2012; Benkler, 2004). In this respect communality, understood as the common ownership of scientific knowledge by all participants, represents a key value that motivates sharing both practices and results. Participant \#14 explains this connection between communality and engagement in relation to practices of another self-researcher:

"He shared his latest thing with RescueTime, where he looked at his apps use and heart rate. I always was interested in stuff like that but right now it is more interesting because I can translate it into action much easier. So I'm also more open and more interested, I guess, in hearing what other people are doing, and seeing if I can pick up cool ideas and incorporate things".

Another Mertonian norm, organized skepticism, for which knowledge generated by science should be open to critical and organized scrutiny, seems to be also present in community presentations and discussions regarding protocol errors, doubts about results, or reliability of tracking tools, even when usually happening in self-organized and unstructured ways. This usually takes place in a context of active and non-judgmental listening, in which the rest of self-trackers provide ideas or feedback but can also 
contribute to the discussion on elaborated technical or intellectual dimensions. As expressed by Participant \#02 about other members of the community:

"They do have enough motivation and skills to start some process of self-management, and then they go into it further and they become these role models. With values of openness and learning and being curious, and being not judgmental as well. I think that's [about] being not judgmental and being open for feedback as well, because it also requires being vulnerable when you give a talk to these other people and they can judge you".

The Mertonian concept of disinterestedness, which in its original formulation stipulates that the "people of science" should act for the benefit of a common scientific enterprise, rather than for personal gain, may seem at odds with the main motivational focus from which personal science projects usually depart - that is, oneself. However, despite originally being motivated by personal research questions or concerns, another of the aspirations several of the interviewees aspire to share as openly as possible their work - in search of objectivity and contrasting findings, but also for the stimulus of other peers benefiting from it. Like expressed by Participant \#16 in contrast to personal motivations:

"There was the aspect of not feeling like that I needed to profit from any of this. And in fact, maybe if other people found and used the things that I made, just the feeling of having that happen was worth more than potentially keeping those things to myself and using them for whatever purpose".

This connects with the fourth Mertonian concept of universalism - a principle of inclusivity for which the acceptance or rejection of truth and validity claims don't depend on the personal or social attributes of the researcher. In line with speculations by Heyen (2016), this ethos seems less evident in personal science beyond the openness that characterises community activities, where such claims are usually implicitly formulated. However, as reflected by Participant \#18 in relation to wider citizen science practices and inclusivity:

"Once you start integrating questions or development of why is this data going like this, you're starting to get engagement with a discussion around a community. I think it starts to become a citizen science activity - and I take a very broad definition of citizen science - in the way I look at a lot of activities being inclusive as long as there's a discourse around the topics that are toward the intellectual stimulation, of kind of refining the ideas or improving upon the process".

Another relevant question that emerged during interviews, when asking participants about how they would define themselves in relation to citizen science is the recurrence of concepts like self-researcher, personal scientist, patient-researcher or independent researcher. Answer from participant \#05 sheds more light on this:

"I much prefer the term personal scientists, because I contrast it with the work of professional scientists. So there are professional scientists and then there are personal scientists. And the professional scientist who does it, does it because that's a job, they do it for a living, whereas a 
personal scientist does it for personal reasons. [...]. The term everyday science also, which I think is another good one".

\section{Conclusion}

Analogous to initial motivations for passive self-tracking (Schüll, 2018), personal science practitioners are moved by the need to solve health conditions or improve well-being, leading to systematic and empirical approaches to acquire personal knowledge. These two types of initial motivations combine over time, evolving in research curiosity and enjoyment through the self-reflexive use of tools, developing new research questions and deductively addressing individual goals.

While extrinsic motivations seem infrequent in self-research, learning by direct practice and by sharing personal progress with peers in community settings represents another key motivational aspect for these practitioners, in line with peer production practices (Spaeth and Niederhöfer, 2020). Additionally, scaling to "N-of-many" beyond N-of-1 approaches by data aggregation (Nafus, 2019) is another of the aspirations identified in our interviews, where participants from patient-led contexts reported to integrate other self-researchers.

Common values observed in personal science - including learning-by-sharing, transparency presenting findings, openness regarding peer's reuse of self-generated data, tools or protocols, and a commitment to discuss collectively self-research approaches - align to a significant extent with traditional scientific principles of communality and organized skepticism, while less with disinterestedness and universalism (Merton, 1973). Overall, these values refer to a more participatory and inclusive scientific culture (Heyen, 2020) driven by self-reflection, critical thinking and collaboration.

The connection between personal science and citizen science (Heyen, 2016), according to the described motivations, goals and values, seems through our study validated and coherent, but more research on such connection is necessary in further studies. Additionally, the iterative stages of the research cycle of personal science (Wolf and De Groot, 2020) often seem not fully formalized nor developed. Further research on learning processes and peer support in self-research should analyse in more detail the evolution of these individual and community practices, identifying barriers, opportunities and advances in this field of practice.

\section{Acknowledgments}

The authors would like to thank all participants of the Open Humans community calls and the Quantified Self community interviewed for this study, as well as the interviewees not active in those communities also for their review and responses regarding our presentation of potentially identifying personal information. The authors are also grateful for the initial suggestions and recommendations of Gary Wolf when framing the initial approach of this study. 


\section{Funding}

The authors disclosed receipt of the following financial support for the research, authorship, and publication of this article: thanks to the Bettencourt Schueller Foundation's long-term partnership, this work was partly supported by the Center for Research and Interdisciplinarity Research Fellowship (awarded to BGT).

\section{Authors' contributions}

ESH and BGT initiated the study. ESH, BGT and MPB developed the study design and protocol. ESH and MO conducted and transcribed the semi-structured interviews. All authors contributed to the coding and interpretation of the interview excerpts. ESH prepared the original draft of the manuscript. ESH, BGT and MPB reviewed and edited the manuscript before submission.

\section{References}

Ajana, B. (2017). Digital Health and the Biopolitics of the Quantified Self. Digital Health, 3(1), 1-18. https://doi.org/10.1177/2055207616689509

Almalki, M., Gray, K., Sanchez, F. M. (2015). The use of self-quantification systems for personal health information: Big data management activities and prospects. Health Information Science and Systems, 3(1), S1. https://doi.org/10.1186/2047-2501-3-S1-S1

Benkler, Y. (2004). Commons-Based Strategies and the Problems of Patents. Science, 305(5687), 1110-1111. https://doi.org/10.1126/science.1100526

Burnside, M., Crocket, H., Mayo, M., Pickering, J., Tappe, A., de Bock, M. (2020). Do-It-Yourself Automated Insulin Delivery: A Leading Example of the Democratization of Medicine. Journal of Diabetes Science and Technology, 14(5), 878-882. https://doi.org/10.1177/1932296819890623

Choe, E. K., Lee, B., Schraefel, m. c. (2015). Characterizing Visualization Insights from Quantified Selfers' Personal Data Presentations. IEEE Computer Graphics and Applications, 35(4), 28-37. https://doi.org/10.1109/MCG.2015.51

Choe, E. K., Lee, N. B., Lee, B., Pratt, W., Kientz, J. A. (2014). Understanding quantified-selfers' practices in collecting and exploring personal data. Proceedings of the SIGCHI Conference on Human Factors in Computing Systems, 1143-1152. https://doi.org/10.1145/2556288.2557372

Chrisinger, B. W. (2020). The Quantified Self-in-Place: Opportunities and Challenges for Place-Based N-of-1 Datasets. Frontiers in Computer Science, 2. https://doi.org/10.3389/fcomp.2020.00038

Christiansen, T. B., Kristensen, D. B., Larsen, J. E. (2018). The 1-Person Laboratory of the Quantified Self Community. In B. Ajana (Ed.), Metric Culture (pp. 97-115). Emerald Publishing Limited. https://doi.org/10.1108/978-1-78743-289-520181006

Couldry, N., Powell, A. (2014). Big Data from the bottom up. Big Data \& Society, 1(2), 2053951714539277. https://doi.org/10.1177/2053951714539277

De Groot, M., Drangsholt, M., Martin-Sanchez, F., Wolf, G. (2017). Single Subject (N-of-1) Research Design, Data Processing, and Personal Science. Methods of Information in Medicine, 56(06), 416-418. https://doi.org/10.3414/ME17-03-0001

Dijk, E. T. K., IJsselsteijn, W. A. (2016). Design beyond the numbers: Sharing, comparing, storytelling and the need for a Quantified Us. Interaction Design and Architecture(s), 2016(29), 121-135.

Dijk, E. T. K., Westerink, J. H. D. M., Beute, F., IJsselsteijn, W. A. (2017). Personal Informatics, Self-Insight, and Behavior Change: A Critical Review of Current Literature. Human-Computer Interaction, 32(5-6), 268-296. https://doi.org/10.1080/07370024.2016.1276456 
Dolejšová, M., Kera, D. (2017). Soylent Diet Self-Experimentation: Design Challenges in Extreme Citizen Science Projects. Proceedings of the 2017 ACM Conference on Computer Supported Cooperative Work and Social Computing, 2112-2123. https://doi.org/10.1145/2998181.2998365

Dulong de Rosnay, M., Le Crosnier, H. (2012). An Introduction to the Digital Commons: From Common-Pool Resources to Community Governance. 1st Thematic IASC Conference on the Knowledge Commons, Belgium. https://dlc.dlib.indiana.edu/dlc/handle/10535/9561

Eikey, E. V., Caldeira, C. M., Figueiredo, M. C., Chen, Y., Borelli, J. L., Mazmanian, M., Zheng, K. (2021). Beyond self-reflection: Introducing the concept of rumination in personal informatics. Personal and Ubiquitous Computing. https://doi.org/10.1007/s00779-021-01573-w

Epstein, D. A., Caldeira, C., Figueiredo, M. C., Lu, X., Silva, L. M., Williams, L., Lee, J. H., Li, Q., Ahuja, S., Chen, Q., Dowlatyari, P., Hilby, C., Sultana, S., Eikey, E. V., Chen, Y. (2020). Mapping and Taking Stock of the Personal Informatics Literature. Proceedings of the ACM on Interactive, Mobile, Wearable and Ubiquitous Technologies, 4(4), 1-38. https://doi.org/10.1145/3432231

Esmonde, K. (2020). 'There's only so much data you can handle in your life': Accommodating and resisting self-surveillance in women's running and fitness tracking practices. Qualitative Research in Sport, Exercise and Health, 12(1), 76-90. https://doi.org/10.1080/2159676X.2019.1617188

Ferretti, F. (2019). Mapping do-it-yourself science. Life Sciences, Society and Policy, 15(1), 1. https://doi.org/10.1186/s40504-018-0090-1

Fricker, M. (2007). Epistemic Injustice: Power and the Ethics of Knowing. Clarendon Press.

Gavrila, V., Garrity, A., Hirschfeld, E., Edwards, B., Lee, J. M. (2019). Peer Support Through a Diabetes Social Media Community. Journal of Diabetes Science and Technology, 13(3), 493-497. https://doi.org/10.1177/1932296818818828

Gimpel, H., Nißen, M., Görlitz, R. (2013). Quantifying the Quantified Self: A Study on the Motivations of Patients to Track Their Own Health. ICIS 2013 Proceedings. https://aisel.aisnet.org/icis2013/proceedings/HealthcareIS/3

Grant, A. D., Wolf, G. I., Nebeker, C. (2019). Approaches to governance of participant-led research: A qualitative case study. BMJ Open, 9(4), e025633. https://doi.org/10.1136/bmjopen-2018-025633

Hecker, S., Haklay, M., bowser, anne, Makuch, Z., Vogel, J., Bonn, A., Gold, M. (2018). Citizen Science - Innovation in Open Science, Society and Policy. https://doi.org/10.14324/111.9781787352339

Heyen, N. B. (2016). Self-Tracking as Knowledge Production: Quantified Self between Prosumption and Citizen Science. In S. Selke (Ed.), Lifelogging: Digital self-tracking and Lifelogging - Between disruptive technology and cultural transformation (pp. 283-301). Springer Fachmedien. https://doi.org/10.1007/978-3-658-13137-1_16

Heyen, N. B. (2020). From self-tracking to self-expertise: The production of self-related knowledge by doing personal science. Public Understanding of Science, 29(2), 124-138. https://doi.org/10.1177/0963662519888757

Heyen, N. B., Dickel, S. (2019). Was ist Personal Health Science? In N. B. Heyen, S. Dickel, A. Brüninghaus (Eds.), Personal Health Science: Persönliches Gesundheitswissen zwischen Selbstsorge und Bürgerforschung (pp. 1-19). Springer Fachmedien. https://doi.org/10.1007/978-3-658-16428-7_1

Jarrahi, M. H., Gafinowitz, N., Shin, G. (2018). Activity trackers, prior motivation, and perceived informational and motivational affordances. Personal and Ubiquitous Computing, 22(2), 433-448. https://doi.org/10.1007/s00779-017-1099-9

Jennett, C., Kloetzer, L., Schneider, D., Iacovides, I., Cox, A., Gold, M., Fuchs, B., Eveleigh, A., Mathieu, K., Ajani, Z., Talsi, Y. (2016). Motivations, learning and creativity in online citizen science. Journal of Science Communication, 15(3), A05. https://doi.org/10.22323/2.15030205

Jethani, S. (2015). Mediating the body: Technology, politics and epistemologies of self. Communication, Politics \& Culture. https://search.informit.org/doi/abs/10.3316/informit.113702521033267

Kaziunas, E., Lindtner, S., Ackerman, M. S., Lee, J. M. (2018). Lived Data: Tinkering With Bodies, Code, and Care Work. Human-Computer Interaction, 33(1), 49-92. 
https://doi.org/10.1080/07370024.2017.1307749

Kempner, J., Bailey, J. (2019). Collective self-experimentation in patient-led research: How online health communities foster innovation. Social Science \& Medicine, 238, 112366. https://doi.org/10.1016/j.socscimed.2019.112366

Kristensen, D. B., Ruckenstein, M. (2018). Co-evolving with self-tracking technologies. New Media \& Society, 20(10), 3624-3640. https://doi.org/10.1177/1461444818755650

Li, I., Dey, A., Forlizzi, J. (2010). A stage-based model of personal informatics systems. In Proceedings of the SIGCHI Conference on Human Factors in Computing Systems (pp. 557-566). Association for Computing Machinery. https://doi.org/10.1145/1753326.1753409

Lupton, D. (2014). Self-Tracking Modes: Reflexive Self-Monitoring and Data Practices (SSRN Scholarly Paper ID 2483549). Social Science Research Network. https://doi.org/10.2139/ssrn.2483549

Lupton, D. (2019). 'It's made me a lot more aware': A new materialist analysis of health self-tracking. Media International Australia, 171(1), 66-79. https://doi.org/10.1177/1329878X19844042

Lupton, D., Smith, G. J. D. (2018). ‘A Much Better Person’: The Agential Capacities of Self-tracking Practices. In B. Ajana (Ed.), Metric Culture (pp. 57-75). Emerald Publishing Limited. https://doi.org/10.1108/978-1-78743-289-520181004

Lyall, B., Robards, B. (2018). Tool, toy and tutor: Subjective experiences of digital self-tracking. Journal of Sociology, 54(1), 108-124. https://doi.org/10.1177/1440783317722854

Marres, N., Stark, D. (2020). Put to the test: For a new sociology of testing. The British Journal of Sociology, 71(3), 423-443. https://doi.org/10.1111/1468-4446.12746

Merton, R. K. (1973). The sociology of science: Theoretical and empirical investigations. University of Chicago Press.

Moore, P., Robinson, A. (2016). The quantified self: What counts in the neoliberal workplace. New Media \& Society, 18(11), 2774-2792. https://doi.org/10.1177/1461444815604328

Munson, S. A., Schroeder, J., Karkar, R., Kientz, J. A., Chung, C.-F., Fogarty, J. (2020). The Importance of Starting With Goals in N-of-1 Studies. Frontiers in Digital Health, 2. https://doi.org/10.3389/fdgth.2020.00003

Nafus, D. (2019). Data Aggregation as Social Relations: Making Datasets from Self-Tracking Data. European Review, 27(3), 440-454. https://doi.org/10.1017/S106279871900005X

Neff, G., Nafus, D. (2016). Self-Tracking. MIT Press.

O'Neil, M., Toupin, S., Pentzold, C. (2020). The Duality of Peer Production. In The Handbook of Peer Production (pp. 1-17). John Wiley \& Sons, Ltd. https://doi.org/10.1002/9781119537151.ch1

Pantzar, M., Ruckenstein, M. (2017). Living the metrics: Self-tracking and situated objectivity. DIGITAL HEALTH, 3, 2055207617712590. https://doi.org/10.1177/2055207617712590

Piras, E. M. (2019). Beyond self-tracking: Exploring and unpacking four emerging labels of patient data work. Health Informatics Journal, 25(3), 598-607. https://doi.org/10.1177/1460458219833121

Rampin, R., Steeves, V., DeMott, S. (2021). Taguette. Zenodo. https://doi.org/10.5281/zenodo.5111814

Rapp, A., Cena, F., Marcengo, A. (2018). Editorial of the Special Issue on Quantified Self and Personal Informatics. Computers, 7(1), 14. https://doi.org/10.3390/computers7010014

Rapp, A., Tirassa, M. (2017). Know Thyself: A Theory of the Self for Personal Informatics. Human-Computer Interaction, 32(5-6), 335-380. https://doi.org/10.1080/07370024.2017.1285704

Régnier, F. (2018). " Goût de liberté » et self-quantification. Reseaux, $n^{\circ}$ 208-209(2), 95-120.

Riggare, S., Hägglund, M., Bredenoord, A. L., de Groot, M., Bloem, B. R. (2021). Ethical Aspects of Personal Science for Persons with Parkinson's Disease: What Happens When Self-Tracking Goes from Selfcare to Publication? Journal of Parkinson's Disease, Preprint(Preprint), 1-7. https://doi.org/10.3233/JPD-212647

Riggare, S., Scott Duncan, T., Hvitfeldt, H., Hägglund, M. (2019). 'You have to know why you're doing this': A mixed methods study of the benefits and burdens of self-tracking in Parkinson's disease. BMC Medical Informatics and Decision Making, 19(1), 175. https://doi.org/10.1186/s12911-019-0896-7 
Roberts, S. (2012). The Growth of Personal Science: Implications for Statistics. 61.

Rooksby, J., Rost, M., Morrison, A., Chalmers, M. (2014). Personal tracking as lived informatics.

Proceedings of the SIGCHI Conference on Human Factors in Computing Systems, 1163-1172. https://doi.org/10.1145/2556288.2557039

Rotman, D., Preece, J., Hammock, J., Procita, K., Hansen, D., Parr, C., Lewis, D., Jacobs, D. (2012). Dynamic changes in motivation in collaborative citizen-science projects. Proceedings of the ACM 2012 Conference on Computer Supported Cooperative Work, 217-226. https://doi.org/10.1145/2145204.2145238

Ruckenstein, M. (2014). Visualized and Interacted Life: Personal Analytics and Engagements with Data Doubles. Societies, 4(1), 68-84. https://doi.org/10.3390/soc4010068

Ruckenstein, M., Pantzar, M. (2017). Beyond the Quantified Self: Thematic exploration of a dataistic paradigm. New Media \& Society, 19(3), 401-418. https://doi.org/10.1177/1461444815609081

Ruckenstein, M., Schüll, N. D. (2017). The Datafication of Health. Annual Review of Anthropology, 46(1), 261-278. https://doi.org/10.1146/annurev-anthro-102116-041244

Schüll, N. D. (2018). Self in the Loop: Bits, Patterns, and Pathways in the Quantified Self. In $A$ Networked Self and Human Augmentics, Artificial Intelligence, Sentience. Routledge.

Sharon, T. (2017). Self-Tracking for Health and the Quantified Self: Re-Articulating Autonomy, Solidarity, and Authenticity in an Age of Personalized Healthcare. Philosophy \& Technology, 30(1), 93-121. https://doi.org/10.1007/s13347-016-0215-5

Sharon, T., Zandbergen, D. (2017). From data fetishism to quantifying selves: Self-tracking practices and the other values of data. New Media \& Society, 19(11), 1695-1709. https://doi.org/10.1177/1461444816636090

Spaeth, S., Niederhöfer, S. (2020). User Motivations in Peer Production. In The Handbook of Peer Production (pp. 123-136). John Wiley \& Sons, Ltd. https://doi.org/10.1002/9781119537151.ch10

Trace, C. B., Zhang, Y. (2019). The quantified-self archive: Documenting lives through self-tracking data. Journal of Documentation, 76(1), 290-316. https://doi.org/10.1108/JD-04-2019-0064

Vayena, E., Brownsword, R., Edwards, S. J., Greshake, B., Kahn, J. P., Ladher, N., Montgomery, J., O’Connor, D., O’Neill, O., Richards, M. P., Rid, A., Sheehan, M., Wicks, P., Tasioulas, J. (2016). Research led by participants: A new social contract for a new kind of research. Journal of Medical Ethics, 42(4), 216-219. https://doi.org/10.1136/medethics-2015-102663

Vuolanto, P., Bergroth, H., Nurmi, J., Salmenniemi, S. (2020). Reconfiguring health knowledges? Contemporary modes of self-care as 'everyday fringe medicine'. Public Understanding of Science, 29(5), 508-523. https://doi.org/10.1177/0963662520934752

Weisse, A. B. (2012). Self-Experimentation and Its Role in Medical Research. Texas Heart Institute Journal, 39(1), 51-54.

West, S., Pateman, R. (2016). Recruiting and Retaining Participants in Citizen Science: What Can Be Learned from the Volunteering Literature? Citizen Science: Theory and Practice, 1(2), 15. https://doi.org/10.5334/cstp.8

Wolf, G. I., De Groot, M. (2020). A Conceptual Framework for Personal Science. Frontiers in Computer Science, 2 . https://doi.org/10.3389/fcomp.2020.00021

Zhao, J., Wang, T., Fan, X. (2015). Patient value co-creation in online health communities: Social identity effects on customer knowledge contributions and membership continuance intentions in online health communities. Journal of Service Management, 26(1), 72-96.

https://doi.org/10.1108/JOSM-12-2013-0344 